\title{
A Green Method for Graphite Exfoliation Using High-Energy Ball Milling.
}

\author{
I. Estrada-Guel ${ }^{1,2}$, F.C. Robles-Hernandez ${ }^{2}$, J.M. Mendoza-Duarte ${ }^{1}$, R. Pérez-Bustamante ${ }^{1}$ and R. \\ Martínez-Sánchez ${ }^{1}$. \\ 1. Centro de Investigación en Materiales Avanzados (CIMAV). Laboratorio Nacional de Nanotecnología \\ Miguel de Cervantes No. 120, 31109, Chihuahua, Chih., Mexico. \\ 2. Department of Mechanical Engineering Technology, University of Houston, Houston, TX 77204- \\ 4020, USA.
}

Graphite, an allotropic and stable form of carbon, is a useful material constituted by multiple layers joined with covalent bonds linked together by a weak Van Der Walls interaction. The single units named graphenes have attracted considerable attention, because of their excellent mechanical, chemical, thermal, electrical properties and low thermal expansion coefficient [1]. The strong covalent $\left(\mathrm{sp}^{2}\right)$ bonds in this unique honeycomb structure of graphene and their atomic scale thickness impart them with these unusual properties [2]. Taking advantage of the weakness of these interactions, it is possible to insert ions, atoms or molecules between the layers in order to obtain graphenes from graphite. In the exfoliation process, elimination of the intercalated species leads to a significant expansion up to hundreds of times along the c-axis, forming a highly porous material. Exfoliated graphite (EG) has been synthesized by galvanic, chemical and thermal treatments of the natural graphite. However, the chemical method is widely used because its simplicity and versatility. Usually EG is produced intercalating acid guest species between the stacked graphene layers via liquid-phase by reaction between graphite and $18 \mathrm{M} \mathrm{H}_{2} \mathrm{SO}_{4}$ in the presence of strong chemical oxidants such as $\mathrm{KMnO}_{4}, \mathrm{HNO}_{3}$ or $\mathrm{H}_{2} \mathrm{O}_{2}$ [3]. However, reaction leftovers are highly toxic and corrosive materials that required careful manipulation and a special confinement.

In the present work, some EG were prepared using a low cost and environmentally friendly mechanochemical route, which avoid the use of highly corrosive and dangerous reagents. Raw materials were: natural graphite and sodium carbonate (a white, odorless powder with an alkaline taste, which is domestically well known for its everyday use as a water softener) and citric acid (a weak organic acid used as a natural preservative/conservative and to add an acidic taste to foods and drinks). Both substances were used in this work as defoliation agents. An equiatomic mixture of sodium carbonate and natural graphite was milled in a SPEX high-energy mill for $1 \mathrm{~h}$. The milled and unmilled mixtures were leached with an aqueous citric acid solution ( $8 \% \mathrm{wt} . / \mathrm{wt}$.), hot refluxed $2 \mathrm{~h}$, washed with distilled water and dried at $80^{\circ} \mathrm{C}$ overnight. Morphological and chemical studies were performed with a scanning electron microscope (JSM5800-LV) and a high-resolution microscope (JSM-7201F).

The Fig. 1 shows some SEM micrographs of graphite $/ \mathrm{Na}_{2} \mathrm{CO}_{3}$ mixtures with 0 and $1 \mathrm{~h}$ of milling where bright zones correspond to high $\mathrm{Na}_{2} \mathrm{CO}_{3}$ concentration regions (black arrows). After milling $\mathrm{Na}_{2} \mathrm{CO}_{3}$ has been dispersed, obtaining a homogeneous mixture. Fig. 2 displays the composition of 1h-milled samples after leaching process. In Fig. 2 can be observed the reduction of $\mathrm{Na}_{2} \mathrm{CO}_{3}$ content after the chemical treatment with citric acid. Note, that aqueous washing solution is composed by sodium citrate, an innocuous substance commonly used as food antioxidant. The Fig. 3 exhibits some SEM micrographs of isolated graphite particles after the leaching process. It is evident the reduction of particle size and an increased level of defoliation with this green process [4]. 


\section{References:}

[1] V. Singh, D. Joung, L. Zhai, Progress in Materials Science 56 (2011) p. 1178-1271.

[2] M. Yang, Y. Houd, N.A. Kotov, Nano Today 7 (2012) p. 430-447.

[3] M. Inagaki, R. Tashiro, Y. Washino, J. Physics and Chemistry of Solids 65 (2004) p. 133-137.

[4] This research was supported by CONACYT (Project No. 169262) and the Redes Temáticas de Nanociencias y Nanotecnología (124886).

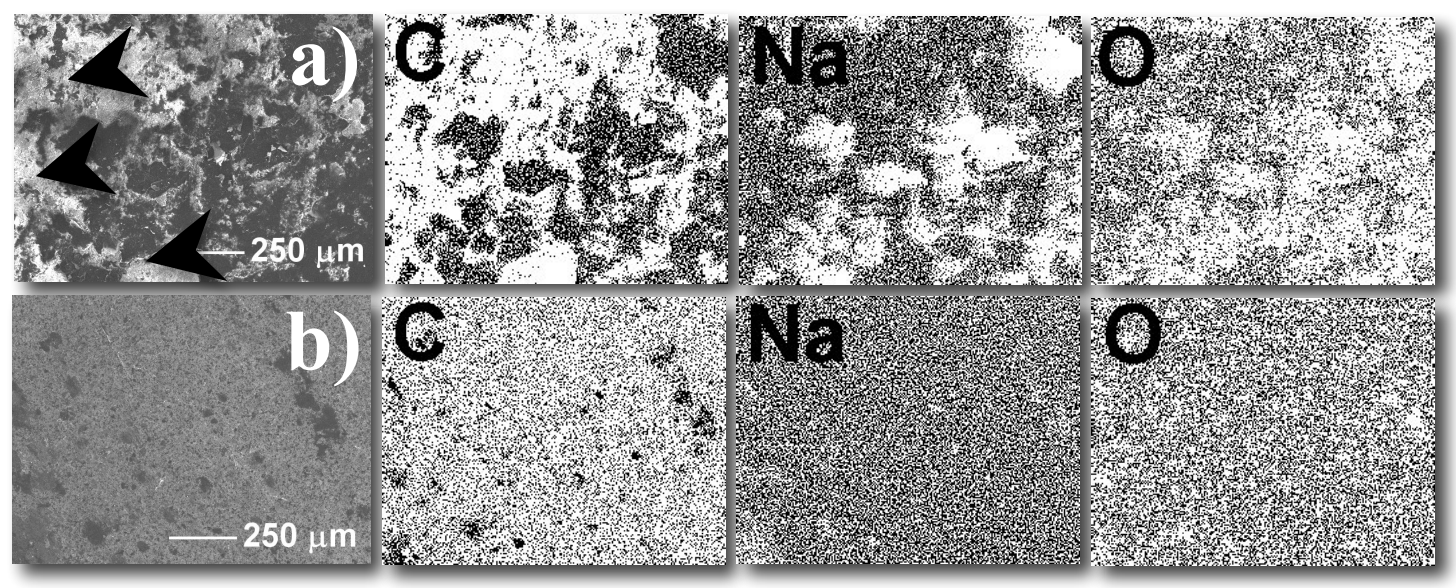

Figure 1. SEM

micrographs and corresponding EDS maps: a) Un-milled mixture $(0 \mathrm{~h})$ and $\mathrm{b})$ sample after $1 \mathrm{~h}$ of milling.
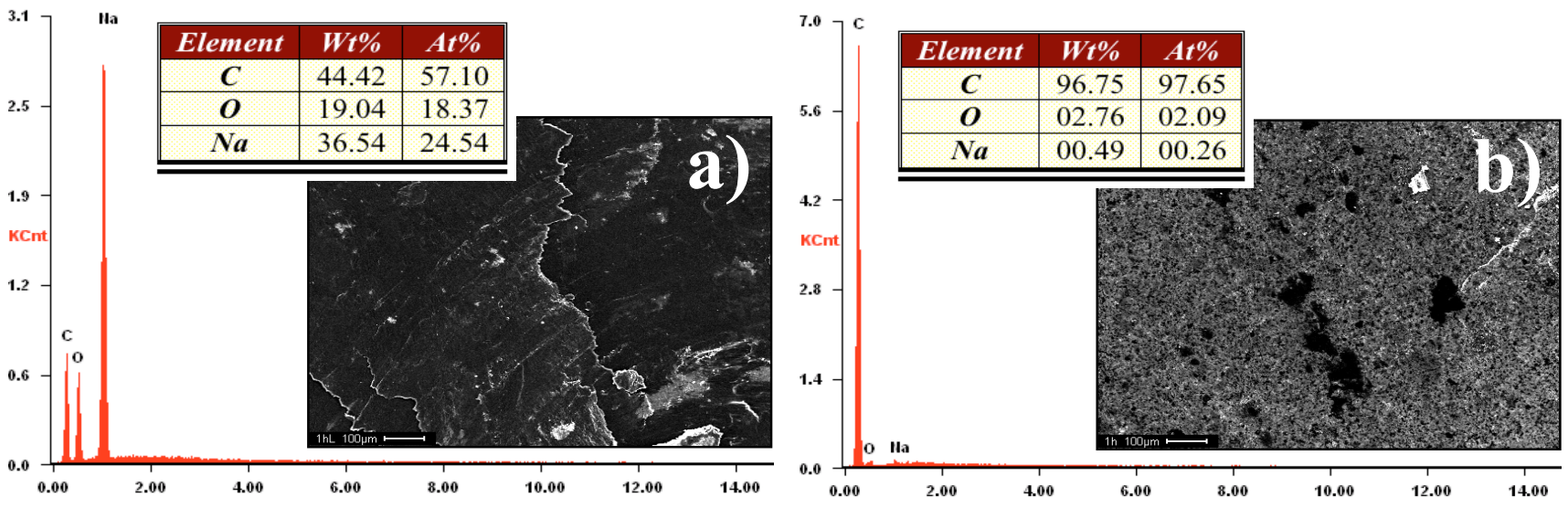

Figure 2. SEM micrographs and EDS elemental analyses: a) $1 \mathrm{~h}$ milled mixture and b) $1 \mathrm{~h}$ leached sample.
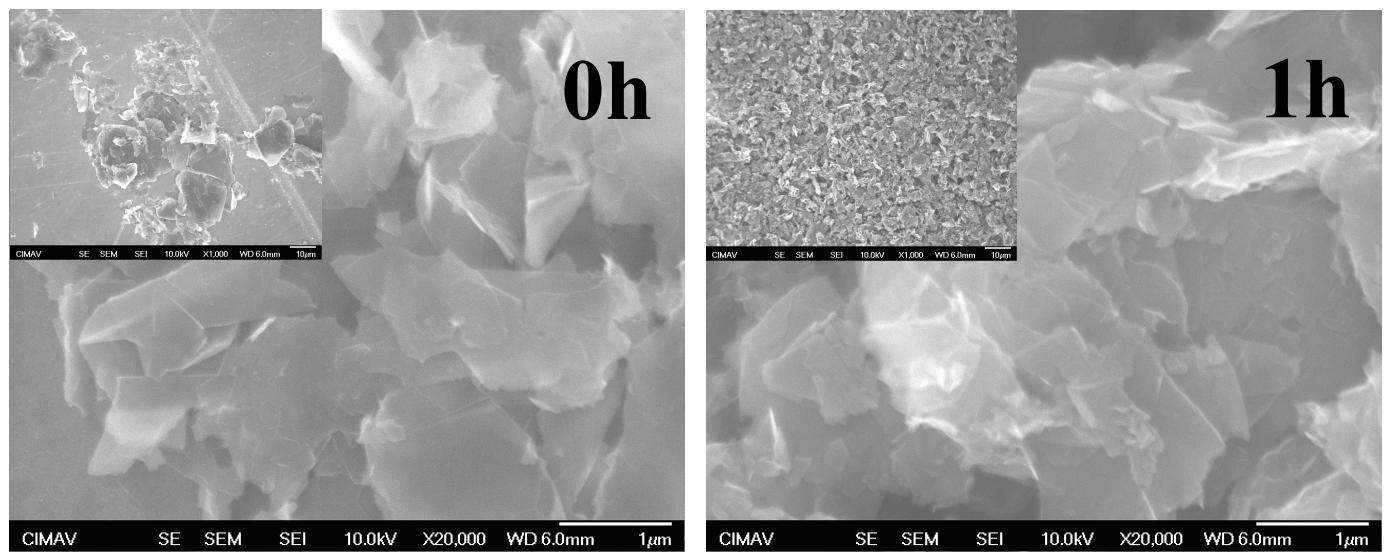

Figure 3. SEM micrographs of graphite particles after the leaching process. 